P97 (continued)

healthy diet as well as foods that should be consumed in moderation. There is a need for HFE assessments that adequately capture a wide spectrum of foods.

Objective: To gain insight into foods that families are serving in the home and to modify the Home Inventory Describing Eating and Activity (Home-IDEA) checklist, a validated instrument for HFE assessment.

Study Design, Setting, Participants, Intervention: Photographs from seven consecutive dinnertime meals lowincome families $(n=29)$ in rural Colorado were used to identify present foods.

Outcomes Measures and Analysis: Foods on the HomeIDEA checklist were dichotomously categorized as healthful (foods that meet the Dietary Guidelines for Americans, i.e. fruits, vegetables, and whole grains) or less healthful (foods high in sodium and solid fats and added sugars, i.e. chips, candy, and soda). Dinnertime photos were qualitatively analyzed to produce a list of foods available in the home environment. This was then compared with the HomeIDEA checklist.

Results: Of 108 foods in the Home-IDEA, 68\% $(n=73)$ represented healthful foods. With the remaining $32 \%$ $(n=35)$ representing less healthful foods. Data analyzed from the photos found 55 foods that were not captured by the Home-IDEA, a majority of which could be considered less healthful, i.e. canned soup, fried taco shells, and prepackaged baked goods.

Conclusions and Implications: In its current state, the Home-IDEA is weighted toward capturing nutrient-rich foods in the HFE. Increased representation of less healthful foods will improve the assessment of HFE quality. HFE assessment tools are strengthened by using a variety of approaches to identify foods for inclusion like meal photography, diet records, or national databases in addition to encompassing dietary guideline recommendations. A mixed methods approach has the potential to yield more comprehensive tools for assessing the quality of the HFE. Funding: NIFA.

\section{P98 Factors that Influence the Efficacy of Nutrition Education Interventions Among Adults: A Systematic Review}

Mary Murimi,PhD,RD, mary.murimi@ttu.edu, Texas Tech University, 1301 Akron Avenue, Lubbock, TX 79409-1270; Bong Nguyen, MS; Michael Kenyi, PhD, Imperial Valley College; Tatenda Mupfudze, PhD, Texas Tech University; M.D. Ruhul Amin, PhD, Dhaka University; Teresia Mbogori, PhD, Ball State University; Khalid Aldubayan, PhD, King Saud University

Background (Background, Rationale, Prior Research, and/or Theory): Efficacy of nutrition education interventions is dependent upon several factors such as duration and frequency of intervention, the study objectives and design. However, it is not clear what factors are more effective in changing behavior.

Objective: To examine systematically factors that contribute to the efficacy of nutrition education interventions among adult population in promoting behavior change for good health based on their stated objective.

Study Design, Setting, Participants, Intervention: This study followed the Preferred Reporting Items for Systematic Reviews and Meta-Analysis (PRISMA) criteria. A total of 246 original articles published between 2009 and 2015 in PubMed, Medline, Web of Science, Academic Search Complete, Science Direct, Cochrane Reviews, ERIC, and PsychLIT were initially considered. The number was screened and scaled down to 40 publications for the final analysis. Quality assessment was based on the Cochcrane Handbook for Systematic Reviews of Intervention.

Outcome Measures and Analysis: The primary analytic goal was to determine the overall effectiveness of nutrition education interventions. Outcome(s) of the study were compared with the stated purpose and/or objectives of the study. Assessed factors included: design, including randomness; type of intervention and activities implemented; duration and dosage; number of objectives; fidelity in implementation; and use of theory. Results: Efficacy of nutrition education interventions depended on major factors: interventions that lasted $\geq 5$ months; having $\leq 3$ focused objectives; appropriate design and use of theories; fidelity; and support from policy makers and management for worksite environmental interventions.

Conclusions and Implications: The results of this review suggest that nutrition education interventions with longer duration, few and focused objectives, assured fidelity, and use of theories have a higher chance of achieving their purpose. For worksite and other environmental interventions, it is important for policy makers to make healthy choices available, for example allowing time for exercise. Researchers should consider including these components when designing future nutrition education interventions for adult populations.

Funding: None.

\section{P99 Improving Vegetable Intake Among Children in Restaurants: The Healthy Bites Pilot Study}

Mackenzie Ferrante, MS, RD, Colorado State University; Jeffrey Miller, PhD; Susan L. Johnson, PhD, University of Colorado, Anschutz Medical Campus;

Laura L. Bellows, PhD, MPH, RD,

laura.bellows@colostate.edu, Colorado State University, Department FSHN, 1571 Campus Delivery, Fort Collins, CO 80523-1571

Background (Background, Rationale, Prior Research, and/or Theory): Children are eating fewer vegetables than currently recommended and families are eating more frequently in restaurants, which is associated with low dietary quality. Novel and innovative strategies are needed to address the nutritional quality of children's meals in restaurants.

Continued on page S55 
P99 (continued)

Objective: To investigate whether offering a vegetable appetizer in a restaurant setting will impact children's vegetable consumption at a meal and whether marketing cues during the appetizer course can further increase vegetable consumption.

Study Design, Setting, Participants, Intervention: Families with at least one child (4-12 years) were recruited from university listservs to attend three dinners serving the same meal (chicken strips, french fries, carrot sticks, ranch dressing, and milk). For Meal 1 children were served all foods simultaneously (control condition). During Meals 2 and 3, the vegetable (carrot sticks) was offered as an appetizer. Meal 3 included marketing prompts highlighting carrots during the appetizer course. Foods offered to children were weighed pre- and post-meal to determine consumption.

Outcome Measures and Analysis: Repeated measures analysis of variance was conducted to determine the effects on carrot consumption (g) with main effects of Meal condition and age (4-7 and 8-12 years).

Results: All families $(n=19)$ participated in three dinners at a university-based restaurant $(n=34$ children, $n=33$ parents). There was a significant effect of Meal condition on carrot consumption $[\mathrm{F}(2,31)=3.50, P=.036]$. Children consumed significantly more carrots (g) during the appetizer conditions compared to the control condition $(13.5 \mathrm{~g}+36.9, P=.04)$. The addition of marketing prompts at Meal 3 did not further increase carrot consumption, $P=.95$. There was a significant main effect for age $[\mathrm{F}(2,31)=3.80, P=.028]$ with younger children demonstrating larger increases in intake $(\overline{\mathrm{x}}=18.7 \mathrm{~g})$ compared to older children $(\overline{\mathrm{x}}=9.0 \mathrm{~g})$.

Conclusions and Impolications: These pilot data suggest that offering a vegetable appetizer may be a feasible strategy to increase children's consumption of vegetables in the restaurant setting, especially for younger children.

Funding: Colorado State University, College of Health and Human Sciences.

\section{P100 Organizational Readiness for Implementing a Nutrition Curriculum in Early Care and Education}

Taren Swindle,PhD, TSwindle@uams.edu, University of Arkansas for Medical Sciences, 521 Jack Stephens Drive \#530, Little Rock, AR 72205-7199;

Leanne Whiteside-Mansell, EdD; Danya Johnson, BS

Background (Background, Rationale, Prior Research, and/or Theory): Preschool educational standards increasingly emphasizes nutrition education as a promising obesity prevention strategy. Success of implementation of new curricula likely vary based on the readiness of the context. An assessment of the readiness of the context may allow implementation to be more successful.

Objective: This study presents results from adaptation of the Organizational Readiness for Implementation Change
(ORIC) instrument to assess educators' readiness to implement the nutrition education curriculum Together, We Inspire Smart Eating (WISE). This study presents psychometric properties of the ORIC in this new setting and assessment of association with perception of barriers.

Study Design, Setting, Participants, Intervention: Educators and administrators $(\mathrm{N}=359)$ completed the ORIC before receiving education on the implementation of WISE. Participants were primarily lead teachers (45\%) with at least some college education (64\%).

Outcome Measures and Analysis: The ORIC instrument ( $\mathrm{N}=12$ items rated on 5-point likert scale) has demonstrated validity and reliability in health care settings. Participants were asked to rate nine potential barriers to implementation.

Results: The reliability for the 12 items was $(\alpha=.97)$. An exploratory factor analyses $(E F A$, eigenvalue $=1$ ) indicated one factor. An EFA constrained to replicate previous work with two factors was not supported. Examination of careless response include split half reliability $(\alpha=.92)$ and an examination of the number of repeating responses (34\% used the same response for all items) suggested respondents did not distinguish items uniquely. However, the validity of the total ORIC was supported by the expected association with the number of barriers perceived by participants $(\mathrm{r}=-30)$.

Conclusions and Implications: The ORIC had high internal consistency and demonstrated convergent validity in this sample. However, indicators of careless responding were elevated suggesting that the ORIC may benefit from a reduction of items. Alternately, it may be possible to present the items in a format that emphasizes the unique contribution of each question.

Funding: NIH, FNS.

\section{P101 Reliability of the Self-Report Home Food Inventory and the Food Preparation Supplies Checklist in College Students}

Abeer Almudaihim, MS, University of Florida; Anne Mathews, PhD, RDN; Melissa Vilaro, PhD; Sarah Colby, PhD, RDN, University of Tennessee; Karla Shelnutt,PhD, RDN, kpagan@ufl.edu, University of Florida, 3028 McCarty Hall D, Gainesville, FL 32611

Background (Background, Rationale, Prior Research, and/or Theory): The home food environment (HFE; food availability and food preparation supplies) appears to be an important mediator of diet quality. The Home Food Inventory (HFI) and the Food Preparation Checklist (FPC) are commonly used tools for assessing HFE but have only been tested via in-home researcher visits, which are time consuming and expensive, but have not been tested via self-report in college students.

Objective: To evaluate the reliability of college students' self-reported HFE using the HFI and the FPC.

Continued on page 556 